\title{
Representations of solutions to linear and bilinear difference equations and systems of bilinear difference equations
}

Stevo Stević $1,2,3^{*}$

*Correspondence: sstevic@ptt.rs

${ }^{1}$ Mathematical Institute of the Serbian Academy of Sciences, Beograd, Serbia

${ }^{2}$ Department of Medical Research,

China Medical University Hospital,

China Medical University, Taichung,

Taiwan, Republic of China

Full list of author information is

available at the end of the article

\section{第 Springer}

\begin{abstract}
We represent general solution to a homogeneous linear difference equation of second order in terms of a specially chosen solution to the equation and apply it to get a representation of general solution to the bilinear difference equation in terms of a solution to an associate difference equation of second order, considerably generalizing some recent results in an elegant way. We also present the corresponding representations for some systems of bilinear difference equations. Many historical notes not so known to wide audience are also presented, and we offer an answer to an open question regarding the attribution of the bilinear difference equation.
\end{abstract}

MSC: Primary 39A05; 39A06

Keywords: Linear difference equation; Bilinear difference equation; General solution; Representation of solutions; System of bilinear difference equations; History of difference equations

\section{Introduction}

It has now been 300 years since the area of difference equations/recurrent relations started to attract a serious interest of scientist working in various branches of science. During these three centuries scientists have obtained many nice results on the equations, and the area has been growing constantly. However, it has been also noticed that more and more researchers interested in the area work on some difference equations and systems without knowing some basic facts on them, so it frequently happens that some basic results are rediscovered and published. Here, among other things, we will demonstrate it on a concrete example of a nonlinear difference equation. One of the aims of the paper, among other ones, is also to give many useful mathematical and historical information to the experts, as well as to everyone who is interested in the area. The reader can find here many old sources, some of which are most probably original ones, which were forgotten during centuries of investigations of recurrent relations, and can see that some known results and formulas are wrongly attributed, a thing which frequently happens.

(c) The Author(s) 2018. This article is distributed under the terms of the Creative Commons Attribution 4.0 International License (http://creativecommons.org/licenses/by/4.0/), which permits unrestricted use, distribution, and reproduction in any medium, provided you give appropriate credit to the original author(s) and the source, provide a link to the Creative Commons license, and indicate if changes were made. 


\subsection{Some history}

Since the time of de Moivre, recurrent relations have been attracting interest of mathematicians. The notion recurrent series/sequence was most probably coined in [1], although such sequences had already appeared in [2]. From [1] and [2] we see that de Moivre had all necessary ingredients for solving homogeneous linear difference equations with constant coefficients in closed form, although some explicit formulas for the solutions to the equations of small orders appeared later in [3] (see also [4]). The results by de Moivre can be regarded as a starting point for a serious investigation of recurrence relations.

There had been few results on recurrent relations before de Moivre, such as Cassini's formula for Fibonacci numbers from 1680, but de Moivre seems to be the first who presented some general methods for dealing with the relations. Of course, it is well known that recurrent relations in some descriptive ways had been already known to Fibonacci [5], and certainly to many other ancient researchers, but important analytic results were not known for a long time.

That recurrent relations can be written in the form of difference equations and viceversa was realized during the eighteenth century by other researchers. Laplace could be the first who noted the connection (see [6]). This is why many researchers have been using both notions interchangeably since that time. This will be also the case in our paper.

Among other ones de Moivre solved the homogeneous second-order linear difference equation with constant coefficients, that is, the following one:

$$
a_{n+1}-a a_{n}-b a_{n-1}=0, \quad n \in \mathbb{N},
$$

where $b \neq 0$, and obtained the following formula:

$$
a_{n}=\frac{a_{1}+(m-a) a_{0}}{m-p} m^{n}+\frac{a_{1}+(p-a) a_{0}}{p-m} p^{n}, \quad n \in \mathbb{N}_{0},
$$

where $m$ and $p$ are the zeros of the polynomial

$$
P_{2, a, b}(t)=t^{2}-a t-b
$$

when $m \neq p$.

He did it in passing, since he was actually interested in finding closed-form formulas for the sums

$$
\sum_{n} \widehat{a}_{n} x^{n}
$$

where the general term $\widehat{a}_{n} x^{n}$ satisfies a relative of equation (1).

A more systematic presentation of the de Moivre methods and ideas can be found in Euler's known book [7], where solutions to some other linear recurrent relations can also be found.

By using Viète's formulas, equality (2) can be written in the following form:

$$
a_{n}=a_{1} \frac{m^{n}-p^{n}}{m-p}+b a_{0} \frac{m^{n-1}-p^{n-1}}{m-p}
$$


for $n \in \mathbb{N}_{0}$.

De Moivre also noticed that if a solution $\left(\widetilde{a}_{n}\right)_{n \in \mathbb{N}_{0}}$ to equation (1) satisfies the following initial conditions:

$$
\widetilde{a}_{0}=1 \text { and } \tilde{a}_{1}=a \text {, }
$$

then

$$
\widetilde{a}_{n}=\frac{m^{n+1}-p^{n+1}}{m-p}
$$

for $n \in \mathbb{N}_{0}$, when $m \neq p$, that is, when $a^{2}+4 b \neq 0$.

Combining (3) and (4) it immediately follows that

$$
a_{n}=a_{1} \widetilde{a}_{n-1}+b a_{0} \widetilde{a}_{n-2}
$$

for $n \geq 2$.

Hence, the representation of solutions to equation (1) given in (5) is a very simple consequence of some results by de Moivre. The simple consequence we could not find in de Moivre's papers, but our observation shows that the representation is/must be a matter of folklore. We have used recently this representation in [8]. The usefulness of solutions (4) has been demonstrated in several recent papers of us on product-type difference equations and systems (see, e.g., $[9,10]$ and the references therein).

If $b=1$, from (5) it is obtained

$$
a_{n}=a_{1} \tilde{a}_{n-1}+a_{0} \tilde{a}_{n-2}
$$

for $n \geq 2$.

If additionally it is assumed that $a=1$, we obtain that representation (6) holds for every solution to the Fibonacci recurrence relation. Moreover, since $\widetilde{a}_{0}=\widetilde{a}_{1}=1$, we have

$$
\widetilde{a}_{n}=f_{n+1}, \quad n \in \mathbb{N}_{0}
$$

where $f_{n}$ is the Fibonacci sequence $[11,12]$.

Hence, from (6) we obtain that in this case

$$
a_{n}=a_{1} f_{n}+a_{0} f_{n-1}, \quad n \in \mathbb{N}_{0},
$$

which is a well-known representation of solutions to the Fibonacci difference equation (see, e.g., [11]).

One of the basic nonlinear difference equations solvable in closed form is

$$
z_{n+1}=\frac{\alpha z_{n}+\beta}{\gamma z_{n}+\delta}, \quad n \in \mathbb{N}_{0}
$$

where $\alpha \delta \neq \beta \gamma, \delta \neq 0$. It is called the bilinear difference equation or linear fractional difference equation (here we use the first name). 
Special cases of equation (8) appeared long time ago since they are connected, for example, to continuous fractions. Note that (8) can be written as follows:

$$
\gamma z_{n+1}+\delta=\alpha+\delta+\frac{\beta \gamma-\alpha \delta}{\gamma z_{n}+\delta}, \quad n \in \mathbb{N}_{0} .
$$

By using the change of variables

$$
w_{n}=\gamma z_{n}+\delta, \quad n \in \mathbb{N}_{0},
$$

the equation becomes

$$
w_{n+1}=\widetilde{\alpha}+\frac{\widetilde{\beta}}{w_{n}}, \quad n \in \mathbb{N}_{0},
$$

where

$$
\widetilde{\alpha}=\alpha+\delta \quad \text { and } \quad \widetilde{\beta}=\beta \gamma-\alpha \delta \text {, }
$$

which defines a continuous fraction.

Since $w_{n}$ is written as a fraction, we may assume that

$$
w_{n}=\frac{x_{n+1}}{y_{n+1}}, \quad n \in \mathbb{N}_{0} .
$$

If it is naturally assumed that

$$
\begin{aligned}
& x_{n+1}=\widetilde{\alpha} x_{n}+\widetilde{\beta} y_{n}, \\
& y_{n+1}=x_{n}
\end{aligned}
$$

for $n \in \mathbb{N}$ (write the right-hand side in (9) as a fraction),

$$
x_{1}=w_{0} \quad \text { and } \quad y_{1}=1 \text {, }
$$

it is easily seen that it must be

$$
w_{n}=\frac{x_{n+1}}{x_{n}}, \quad n \in \mathbb{N}_{0}
$$

(note that from (10) and (11) with $n=0$ it is obtained that $x_{0}=1$ ). These and many other data on various continuous fractions can be found in several papers in [13], see also [1416].

If in (9) we take the change of variables

$$
\widetilde{w}_{n}=\frac{1}{w_{n}}, \quad n \in \mathbb{N}_{0}
$$

then it is obtained

$$
\widetilde{w}_{n+1}=\frac{1}{\widetilde{\alpha}+\widetilde{\beta} \widetilde{w}_{n}}, \quad n \in \mathbb{N}_{0},
$$


and from the above consideration we have

$$
\widetilde{w}_{n}=\frac{x_{n}}{x_{n+1}}, \quad n \in \mathbb{N}_{0}
$$

This is an explanation why equation (8) is usually solved by using the change of variables

$$
z_{n}=\frac{1}{\gamma}\left(\frac{x_{n+1}}{x_{n}}-\delta\right), \quad n \in \mathbb{N}_{0},
$$

and why the generalized bilinear difference equation

$$
z_{n+1}=\frac{\alpha_{n} z_{n}+\beta_{n}}{\gamma_{n} z_{n}+\delta_{n}}, \quad n \in \mathbb{N}_{0},
$$

is transformed to a linear second-order difference equation by a similar change of variables.

The change of variables can already be found in papers by Laplace, see, e.g., [6]. Hence, the use of the change of variables is a common method which has been applied since (see, e.g., [17-27]). For a different approach in solving equation (8), which uses a linear system of difference equations, see [15, 28]. A related method has been recently used in [29] for solving another system of difference equations. See also [30]. Methods for solving some linear systems of difference equations appeared also in [6]. We have to mention that both methods transform equation (8) to an equation of the form (1). For some applications of equation (8), see also [31-33].

If $\gamma=0$, then equation (8) is reduced to the following linear first-order difference equation:

$$
z_{n+1}=\tilde{a} z_{n}+\widetilde{b}, \quad n \in \mathbb{N}_{0}
$$

where

$$
\widetilde{a}=\frac{\alpha}{\delta} \quad \text { and } \quad \tilde{b}=\frac{\beta}{\delta}
$$

General solution to equation (15) is

$$
\begin{aligned}
& z_{n}=\widetilde{a}^{n}\left(z_{0}+\frac{\widetilde{b}}{\widetilde{a}-1}\right)+\frac{\widetilde{b}}{1-\widetilde{a}}, \quad \text { when } \widetilde{a} \neq 1 ; \\
& z_{n}=z_{0}+n \widetilde{b}, \quad \text { when } \widetilde{a}=1,
\end{aligned}
$$

and can be found in [34].

Equation (15) is a special case of the nonhomogeneous linear first-order difference equation with variable coefficients

$$
z_{n+1}=a_{n} z_{n}+b_{n}, \quad n \in \mathbb{N}_{0},
$$

which was also solved by Lagrange in [34]. Another method for solving equation (15) can be found in Laplace's paper [6]. 
There are three standard methods for solving equation (18) which correspond to the three methods for solving the linear differential equation of first order. A presentation of the methods can be found in [26]. Many books on difference equations which deal with equation (18) solve it by using one of the methods [15, 20, 22-24, 35]. Usefulness of the equation in solving many classes of nonlinear difference equations and systems has been recently demonstrated in many papers [32,36-42] (see also the references therein; see also $[43,44]$ and compare the methods therein with the solvability ones). For some applications and qualitative analysis of the solutions to equation (18), see [23, 45-47].

Regarding our investigations, we will only say here that our renewed considerable interest, which essentially always existed to some extent, in solvability of difference equations started about fifteen years ago when we noted that the following nonlinear difference equation

$$
x_{n+1}=\frac{x_{n-1}}{a+b x_{n} x_{n-1}}, \quad n \in \mathbb{N}_{0},
$$

can be solved in closed form using a change of variables, which transforms equation (19) to a special case of equation (18), showing a relative simplicity of the equation. Because of the simplicity we did not expect a special interest in it. However, a bit surprisingly, the method has attracted a considerable interest of researchers and many of them started employing our ideas, which consequently motivated us to study solvability of extensions of equation (19) such as those in [37,38], and [41], as well as of some related systems of difference equation [40,42]. Some of our recent methods, ideas, and new classes of solvable equations and systems can be found, for example, in [10] and [39]; see also the references therein.

For some other solvable difference equations and systems or the ones which have invariant integrals and their applications, see, e.g., [48-58] and the references therein.

\subsection{On a representation of an equation of form (8)}

It is easy to check that solution (4) to equation (1) holds for every $n \in \mathbb{Z}$, and that $a_{-1}=0$. Hence, the solution is the one with initial conditions $a_{-1}=0$ and $a_{0}=1$. As we have already mentioned, the Fibonacci sequence is of this type, but with shifted indices (recall that $f_{0}=0$ and $f_{1}=1$ ). This was one of the motivations for us to introduce in [8] the generalized Fibonacci sequences as those solutions to equation (1) satisfying the initial conditions

$$
a_{0}=0 \quad \text { and } \quad a_{1}=1
$$

Our idea in [8] was to generalize some representations to solutions to equations (1) and (8) which are given in terms of the Fibonacci sequence.

One of our motivations was the following representation:

$$
z_{n}=\frac{z_{0} f_{n-1}+f_{n}}{z_{0} f_{n}+f_{n+1}}
$$

of well-defined solutions to the following difference equation:

$$
z_{n+1}=\frac{1}{1+z_{n}}, \quad n \in \mathbb{N}_{0}
$$

which can be found in [59] and was not theoretically explained there. 
Equation (22) is one of the most known difference equations and can be found in many books dealing with sequences or problem books (see, e.g., [60]). Some other special cases of equation (8) can be also found in [61]. Moreover, formula (21) immediately follows from very old results and is essentially well known. Namely, as explained above, equation (22) is solved by using the change of variables (13), known to Laplace already [6], and the following is obtained:

$$
x_{n+2}=x_{n+1}+x_{n}, \quad n \in \mathbb{N}_{0} \text {. }
$$

By the known representation (7) [11], essentially known to de Moivre [3], we have

$$
x_{n}=x_{0} f_{n-1}+x_{1} f_{n}, \quad n \in \mathbb{N}_{0},
$$

and consequently

$$
z_{n}=\frac{x_{n}}{x_{n+1}}=\frac{x_{0} f_{n-1}+x_{1} f_{n}}{x_{0} f_{n}+x_{1} f_{n+1}}=\frac{z_{0} f_{n-1}+f_{n}}{z_{0} f_{n}+f_{n+1}}
$$

for $n \in \mathbb{N}_{0}$.

\subsection{On a recent extension of representation (21)}

In [8] we generalized representation formula (21) for the case of solutions to general equation (8) by proving the following result.

Theorem 1 Consider equation (8), with $\gamma \neq 0$ and

$$
\alpha \delta \neq \beta \gamma
$$

Let $\left(s_{n}\right)_{n \in \mathbb{N}_{0}}$ be the solution to equation

$$
c_{n+1}-(\alpha+\delta) c_{n}+(\alpha \delta-\beta \gamma) c_{n-1}=0, \quad n \in \mathbb{N},
$$

satisfying the initial conditions

$$
c_{0}=0 \quad \text { and } \quad c_{1}=1
$$

Then every well-defined solution to equation (8) has the following representation:

$$
z_{n}=\frac{(\beta \gamma-\alpha \delta) z_{0} s_{n-1}+\left(\alpha z_{0}+\beta\right) s_{n}}{\left(\gamma z_{0}-\alpha\right) s_{n}+s_{n+1}}
$$

for $n \in \mathbb{N}_{0}$.

Using formulas (16) and (17) and some calculations it is easily proved that Theorem 1 also holds in the case when $\gamma=0$ [62]. Bearing in mind this fact we see that the following somewhat more general result holds. 
Theorem 2 Consider equation (8). Let condition (25) hold and let $\left(s_{n}\right)_{n \in \mathbb{N}_{0}}$ be the solution to equation (26) satisfying the initial conditions in (27). Then every well-defined solution to equation (8) has representation (28).

\subsection{Problems treated in the paper}

Although the solution to equation (1) satisfying initial conditions (20) enables a representation of all the solutions to the equation in a nice form, it is a natural question if some of the other solutions to the equation can also represent all the solutions in a similar form, and if so, which ones of them can do this. More precisely, the problem is to find all $\vec{v}=\left(v_{0}, v_{1}\right) \in \mathbb{R}^{2}$ (or $\left.\in \mathbb{C}^{2}\right)$ such that for every solution $\left(x_{n}\right)_{n \in \mathbb{N}_{0}}$ to the equation the following representation holds:

$$
x_{n}=c_{1} s_{n}(\vec{v})+c_{2} s_{n-1}(\vec{v}), \quad n \in \mathbb{N}_{0},
$$

for some constants $c_{j}, j=1,2$, depending on $\vec{v}, x_{0}$, and $x_{1}$.

Note here that since $b \neq 0$, for every solution $\left(x_{n}\right)_{n \in \mathbb{N}_{0}}$ to equation (1) the value $x_{-1}$ can be naturally defined by using the recurrent relation (1) for $n=0$, as

$$
x_{-1}=\frac{x_{1}-a x_{0}}{b} .
$$

Another aim of ours here is to give a positive answer to the question and to apply the obtained result in getting representations of solutions to equation (8) as well as to the corresponding representations for some bilinear systems of difference equations. Besides, we offer here an answer to an open problem circulating among some experts regarding the attribution of equation (8).

\section{General representation of solutions to equation (8)}

In this section we prove our main results. The first one is a generalization of Theorem 2.

Theorem 3 Let $\vec{v}=\left(v_{0}, v_{1}\right) \in \mathbb{R}^{2}\left(\right.$ or $\left.\in \mathbb{C}^{2}\right)$ and $\left(s_{n}(\vec{v})\right)_{n \in \mathbb{N}_{0}}$ be the solution to equation (1) such that

$$
s_{0}=v_{0} \quad \text { and } \quad s_{1}=v_{1}
$$

Then all the solutions to equation (1) have representation (29) if and only if

$$
b v_{0}^{2}+a v_{0} v_{1} \neq v_{1}^{2}
$$

Further, if (31) holds, then for every solution $\left(x_{n}\right)_{n \in \mathbb{N}_{0}}$ to equation (1) the following representation holds:

$$
x_{n}=\frac{\left(b v_{0} x_{0}+\left(a v_{0}-v_{1}\right) x_{1}\right) s_{n}(\vec{v})+b\left(v_{0} x_{1}-v_{1} x_{0}\right) s_{n-1}(\vec{v})}{b v_{0}^{2}+a v_{0} v_{1}-v_{1}^{2}}
$$

for $n \in \mathbb{N}_{0}$. 
Proof Since $s_{n}(\vec{v})$ is a solution to linear equation (1) so is every sequence of the following form:

$$
c_{1} s_{n}(\vec{v})+c_{2} s_{n-1}(\vec{v}), \quad n \in \mathbb{N}_{0},
$$

where $c_{1}$ and $c_{2}$ are arbitrary constants.

This means that the set

$$
S:=\left\{c_{1} s_{n}(\vec{v})+c_{2} s_{n-1}(\vec{v}): c_{j} \in \mathbb{R}(\text { or } \in \mathbb{C}), j=1,2\right\}
$$

is a subset of the set of all solutions to equation (1). Now we prove that these two sets are equal if and only if condition (31) is satisfied.

Since each solution $\left(x_{n}\right)_{n \in \mathbb{N}_{0}}$ to equation (1) is completely determined by its initial values $x_{0}$ and $x_{1}$, we see that (29) holds for every solution $\left(x_{n}\right)_{n \in \mathbb{N}_{0}}$ to the equation if and only if, for every $\left(x_{0}, x_{1}\right) \in \mathbb{R}^{2}\left(\right.$ or $\left.\in \mathbb{C}^{2}\right)$,

$$
\begin{aligned}
& x_{0}=c_{1} s_{0}(\vec{v})+c_{2} s_{-1}(\vec{v}), \\
& x_{1}=c_{1} s_{1}(\vec{v})+c_{2} s_{0}(\vec{v})
\end{aligned}
$$

for some constants $c_{1}$ and $c_{2}$.

Due to (30), this means that (29) holds for every solution to the equation if and only if the linear system

$$
\begin{aligned}
& v_{0} c_{1}+v_{-1} c_{2}=x_{0} \\
& v_{1} c_{1}+v_{0} c_{2}=x_{1}
\end{aligned}
$$

in variables $c_{1}$ and $c_{2}$ has a solution for every $\left(x_{0}, x_{1}\right) \in \mathbb{R}^{2}$ (or $\in \mathbb{C}^{2}$ ).

This will happen if and only if the determinant of system (33) is different from zero, that is, if and only if

$$
\Delta:=\left|\begin{array}{cc}
v_{0} & v_{-1} \\
v_{1} & v_{0}
\end{array}\right|=v_{0}^{2}-v_{1} v_{-1}=\frac{b v_{0}^{2}+a v_{0} v_{1}-v_{1}^{2}}{b} \neq 0,
$$

from which the first part of the theorem follows.

Now assume that (31) holds. Then from (33) it follows that

$$
c_{1}=\frac{1}{\Delta}\left|\begin{array}{cc}
x_{0} & \left(v_{1}-a v_{0}\right) / b \\
x_{1} & v_{0}
\end{array}\right|=\frac{b v_{0} x_{0}+\left(a v_{0}-v_{1}\right) x_{1}}{b v_{0}^{2}+a v_{0} v_{1}-v_{1}^{2}}
$$

and

$$
c_{2}=\frac{1}{\Delta}\left|\begin{array}{cc}
v_{0} & x_{0} \\
v_{1} & x_{1}
\end{array}\right|=\frac{b\left(v_{0} x_{1}-v_{1} x_{0}\right)}{b v_{0}^{2}+a v_{0} v_{1}-v_{1}^{2}} .
$$

Using (35) and (36) in (29), we get (32), as claimed. 
Remark 1 The set $\widetilde{S}$ consisting of all $\vec{v} \in \mathbb{C}^{2}$ such that (31) does not hold consists of the zero vector $\overrightarrow{0} \in \mathbb{C}^{2}$, and of those $\vec{v}$ such that

$$
\left(\frac{v_{1}}{v_{0}}\right)^{2}-a \frac{v_{1}}{v_{0}}-b=0
$$

that is,

$$
v_{1}=\frac{a+\sqrt{a^{2}+4 b}}{2} v_{0}
$$

or

$$
v_{1}=\frac{a-\sqrt{a^{2}+4 b}}{2} v_{0}
$$

so

$$
\widetilde{S}=\left\{\left(v_{0}, \frac{a \pm \sqrt{a^{2}+4 b}}{2} v_{0}\right): v_{0} \in \mathbb{C}\right\}
$$

By representation (32) a generalization of the representation of solutions to difference equation (8) given in (28) can be obtained. Namely, the following theorem holds.

Theorem 4 Consider equation (8) with $\alpha \delta \neq \beta \gamma$. Assume that $\vec{v}=\left(v_{0}, v_{1}\right) \in \mathbb{R}^{2}$ (or $\left.\in \mathbb{C}^{2}\right)$ satisfies the following condition:

$$
(\beta \gamma-\alpha \delta) v_{0}^{2}+(\alpha+\delta) v_{0} v_{1} \neq v_{1}^{2}
$$

Let $\left(s_{n}\right)_{n \in \mathbb{N}_{0}}$ be the solution to equation (26) such that $s_{0}=v_{0}$ and $s_{1}=v_{1}$. Then every welldefined solution $\left(z_{n}\right)_{n \in \mathbb{N}_{0}}$ to equation (8) has the following representation:

$$
z_{n}=\frac{(\beta \gamma-\alpha \delta)\left(\beta v_{0}+\left(\alpha v_{0}-v_{1}\right) z_{0}\right) s_{n-1}+\left(\beta\left((\alpha+\delta) v_{0}-v_{1}\right)+z_{0}\left(v_{0}\left(\beta \gamma+\alpha^{2}\right)-\alpha v_{1}\right)\right) s_{n}}{\left((\beta \gamma-\alpha \delta) v_{0}+\alpha v_{1}-\gamma v_{1} z_{0}\right) s_{n}+\left(\delta v_{0}-v_{1}+\gamma v_{0} z_{0}\right) s_{n+1}}
$$

for $n \in \mathbb{N}_{0}$.

Proof First assume that $\gamma \neq 0$. Then from (14) we see that for every well-defined solution $\left(z_{n}\right)_{n \in \mathbb{N}_{0}}$ to equation (8) the following holds:

$$
z_{n}=\frac{1}{\gamma}\left(\frac{x_{n+1}}{x_{n}}-\delta\right), \quad n \in \mathbb{N}_{0}
$$

where $x_{n}$ is a solution to equation (26).

On the other hand, since (37) holds, from Theorem 3 we have

$$
x_{n}=\frac{\left((\beta \gamma-\alpha \delta) v_{0} x_{0}+\left((\alpha+\delta) v_{0}-v_{1}\right) x_{1}\right) s_{n}+(\beta \gamma-\alpha \delta)\left(v_{0} x_{1}-v_{1} x_{0}\right) s_{n-1}}{(\beta \gamma-\alpha \delta) v_{0}^{2}+(\alpha+\delta) v_{0} v_{1}-v_{1}^{2}}
$$

for every solution to equation (26). 
From (39), (40), after some standard but time-consuming calculations and use of (26), we have

$$
\begin{aligned}
& z_{n}=\frac{1}{\gamma}\left(\frac{\left((\beta \gamma-\alpha \delta) v_{0} x_{0}+\left((\alpha+\delta) v_{0}-v_{1}\right) x_{1}\right) s_{n+1}+(\beta \gamma-\alpha \delta)\left(v_{0} x_{1}-v_{1} x_{0}\right) s_{n}}{\left((\beta \gamma-\alpha \delta) v_{0} x_{0}+\left((\alpha+\delta) v_{0}-v_{1}\right) x_{1}\right) s_{n}+(\beta \gamma-\alpha \delta)\left(v_{0} x_{1}-v_{1} x_{0}\right) s_{n-1}}-\delta\right) \\
& =\frac{1}{\gamma} \frac{\left((\beta \gamma-\alpha \delta) v_{0}+\left((\alpha+\delta) v_{0}-v_{1}\right)\left(\gamma z_{0}+\delta\right)\right)\left(\alpha s_{n}+(\beta \gamma-\alpha \delta) s_{n-1}\right)}{\left((\beta \gamma-\alpha \delta) v_{0}+\left((\alpha+\delta) v_{0}-v_{1}\right)\left(\gamma z_{0}+\delta\right)\right) s_{n}+\left(v_{0}\left(\gamma z_{0}+\delta\right)-v_{1}\right)\left(s_{n+1}-(\alpha+\delta) s_{n}\right)} \\
& +\frac{1}{\gamma} \frac{(\beta \gamma-\alpha \delta)\left(v_{0}\left(\gamma z_{0}+\delta\right)-v_{1}\right)\left(s_{n}-\delta s_{n-1}\right)}{\left((\beta \gamma-\alpha \delta) v_{0}+\left((\alpha+\delta) v_{0}-v_{1}\right)\left(\gamma z_{0}+\delta\right)\right) s_{n}+\left(v_{0}\left(\gamma z_{0}+\delta\right)-v_{1}\right)\left(s_{n+1}-(\alpha+\delta) s_{n}\right)} \\
& =\frac{(\beta \gamma-\alpha \delta)\left(\beta v_{0}+\left(\alpha v_{0}-v_{1}\right) z_{0}\right) s_{n-1}+\left(\beta\left((\alpha+\delta) v_{0}-v_{1}\right)+z_{0}\left(v_{0}\left(\beta \gamma+\alpha^{2}\right)-\alpha v_{1}\right)\right) s_{n}}{\left((\beta \gamma-\alpha \delta) v_{0}+\alpha v_{1}-\gamma v_{1} z_{0}\right) s_{n}+\left(\delta v_{0}-v_{1}+\gamma v_{0} z_{0}\right) s_{n+1}},
\end{aligned}
$$

finishing the proof, in this case.

Now assume that $\gamma=0$. Then equation (8) becomes

$$
z_{n+1}=\frac{\alpha}{\delta} z_{n}+\frac{\beta}{\delta}, \quad n \in \mathbb{N}_{0}
$$

(note that since $\alpha \delta \neq \beta \gamma=0, \delta$ cannot be equal to zero so that equation (41) is defined).

From (16) and (17), it follows that

$$
\begin{aligned}
& z_{n}=\left(\frac{\alpha}{\delta}\right)^{n}\left(z_{0}+\frac{\beta}{\alpha-\delta}\right)-\frac{\beta}{\alpha-\delta}, \quad \text { when } \alpha \neq \delta, \\
& z_{n}=z_{0}+\frac{\beta}{\delta} n, \quad \text { when } \alpha=\delta
\end{aligned}
$$

for $n \in \mathbb{N}_{0}$.

Let the right-hand side in (38) with $\gamma=0$ be denoted by $\tilde{z}_{n}$. Then we have

$$
\tilde{z}_{n}:=\frac{\alpha \delta\left(\beta v_{0}+\left(\alpha v_{0}-v_{1}\right) z_{0}\right) s_{n-1}-\left(\beta\left((\alpha+\delta) v_{0}-v_{1}\right)+\alpha\left(\alpha v_{0}-v_{1}\right) z_{0}\right) s_{n}}{\alpha\left(\delta v_{0}-v_{1}\right) s_{n}+\left(v_{1}-\delta v_{0}\right) s_{n+1}}
$$

for $n \in \mathbb{N}_{0}$.

By de Moivre's formula (2) we know that

$$
s_{n}=\frac{\left(\delta v_{0}-v_{1}\right) \alpha^{n}+\left(v_{1}-\alpha v_{0}\right) \delta^{n}}{\delta-\alpha}, \quad n \in \mathbb{N}_{0}
$$

if $\alpha \neq \delta$, while

$$
s_{n}=\left(v_{1} n+\alpha v_{0}(1-n)\right) \alpha^{n-1}, \quad n \in \mathbb{N}_{0},
$$

if $\alpha=\delta$, since $\alpha$ and $\delta$ are the zeros of the characteristic polynomial

$$
P_{2}(\lambda)=\lambda^{2}-(\alpha+\delta) \lambda+\alpha \delta
$$

associated to equation (26) in the case $\gamma=0$. 
Assume that $\alpha \neq \delta$. Then, by using (45) in (44), after some calculation we obtain

$$
\begin{aligned}
\tilde{z}_{n}= & \frac{\alpha \delta\left(\beta v_{0}+\left(\alpha v_{0}-v_{1}\right) z_{0}\right)\left(\left(\delta v_{0}-v_{1}\right) \alpha^{n-1}+\left(v_{1}-\alpha v_{0}\right) \delta^{n-1}\right)}{\alpha\left(\delta v_{0}-v_{1}\right)\left(\left(\delta v_{0}-v_{1}\right) \alpha^{n}+\left(v_{1}-\alpha v_{0}\right) \delta^{n}\right)+\left(v_{1}-\delta v_{0}\right)\left(\left(\delta v_{0}-v_{1}\right) \alpha^{n+1}+\left(v_{1}-\alpha v_{0}\right) \delta^{n+1}\right)} \\
& -\frac{\left(\beta\left((\alpha+\delta) v_{0}-v_{1}\right)+\alpha\left(\alpha v_{0}-v_{1}\right) z_{0}\right)\left(\left(\delta v_{0}-v_{1}\right) \alpha^{n}+\left(v_{1}-\alpha v_{0}\right) \delta^{n}\right)}{\alpha\left(\delta v_{0}-v_{1}\right)\left(\left(\delta v_{0}-v_{1}\right) \alpha^{n}+\left(v_{1}-\alpha v_{0}\right) \delta^{n}\right)+\left(v_{1}-\delta v_{0}\right)\left(\left(\delta v_{0}-v_{1}\right) \alpha^{n+1}+\left(v_{1}-\alpha v_{0}\right) \delta^{n+1}\right)} \\
= & \frac{\left(v_{1}-\alpha v_{0}\right)\left(v_{1}-\delta v_{0}\right)\left(\beta \delta^{n}+\left(-\beta+(\delta-\alpha) z_{0}\right) \alpha^{n}\right)}{\left(v_{1}-\alpha v_{0}\right)\left(v_{1}-\delta v_{0}\right)(\delta-\alpha) \delta^{n}}
\end{aligned}
$$

Because of (37) it must be

$$
v_{1} \neq \alpha v_{0} \quad \text { and } \quad v_{1} \neq \delta v_{0} .
$$

From (48) and since $\alpha \neq \delta$, from (47) we obtain

$$
\tilde{z}_{n}=\frac{\beta}{\delta-\alpha}+\left(-\frac{\beta}{\delta-\alpha}+z_{0}\right)\left(\frac{\alpha}{\delta}\right)^{n} .
$$

From (42) and (49) we see that

$$
\tilde{z}_{n}=z_{n}, \quad n \in \mathbb{N}_{0},
$$

from which the result follows in this case.

If $\alpha=\delta$, then by using the assumption and (46) in (44), after some calculation we obtain

$$
\begin{aligned}
\tilde{z}_{n}= & \frac{\alpha \delta\left(\beta v_{0}+\left(\alpha v_{0}-v_{1}\right) z_{0}\right)\left(\left(v_{1}-\alpha v_{0}\right)(n-1)+\alpha v_{0}\right) \alpha^{n-2}}{\alpha\left(\delta v_{0}-v_{1}\right)\left(\left(v_{1}-\alpha v_{0}\right) n+\alpha v_{0}\right) \alpha^{n-1}+\left(v_{1}-\delta v_{0}\right)\left(\left(v_{1}-\alpha v_{0}\right)(n+1)+\alpha v_{0}\right) \alpha^{n}} \\
& -\frac{\left(\beta\left((\alpha+\delta) v_{0}-v_{1}\right)+\alpha\left(\alpha v_{0}-v_{1}\right) z_{0}\right)\left(\left(v_{1}-\alpha v_{0}\right) n+\alpha v_{0}\right) \alpha^{n-1}}{\alpha\left(\delta v_{0}-v_{1}\right)\left(\left(v_{1}-\alpha v_{0}\right) n+\alpha v_{0}\right) \alpha^{n-1}+\left(v_{1}-\delta v_{0}\right)\left(\left(v_{1}-\alpha v_{0}\right)(n+1)+\alpha v_{0}\right) \alpha^{n}} \\
= & \frac{\left(\beta v_{0}+\left(\alpha v_{0}-v_{1}\right) z_{0}\right)\left(\left(v_{1}-\alpha v_{0}\right) n+2 \alpha v_{0}-v_{1}\right) \alpha^{n}}{\left(\left(\alpha v_{0}-v_{1}\right)\left(\left(v_{1}-\alpha v_{0}\right) n+\alpha v_{0}\right)+\left(v_{1}-\alpha v_{0}\right)\left(\left(v_{1}-\alpha v_{0}\right) n+v_{1}\right)\right) \alpha^{n}} \\
& -\frac{\left(\beta\left(2 \alpha v_{0}-v_{1}\right)+\alpha\left(\alpha v_{0}-v_{1}\right) z_{0}\right)\left(\left(v_{1}-\alpha v_{0}\right) n+\alpha v_{0}\right) \alpha^{n-1}}{\left(\left(\alpha v_{0}-v_{1}\right)\left(\left(v_{1}-\alpha v_{0}\right) n+\alpha v_{0}\right)+\left(v_{1}-\alpha v_{0}\right)\left(\left(v_{1}-\alpha v_{0}\right) n+v_{1}\right)\right) \alpha^{n}} \\
= & \frac{\left(v_{1}-\alpha v_{0}\right)^{2}\left(\beta n+\alpha z_{0}\right) \alpha^{n-1}}{\left(v_{1}-\alpha v_{0}\right)^{2} \alpha^{n}}
\end{aligned}
$$

for $n \in \mathbb{N}_{0}$.

Now note that due to (37) it must be

$$
v_{1} \neq \alpha v_{0} .
$$

From (52) and since $\alpha \neq 0$, from (51) we obtain

$$
\tilde{z}_{n}=\frac{\beta n+\alpha z_{0}}{\alpha}
$$

for $\in \mathbb{N}_{0}$.

From (43) and (53) we see that (50) holds also in this case, finishing the proof of the theorem. 


\subsection{On a bilinear system of difference equations}

It is a natural problem to see if there is a representation similar to the one in Theorem 4 for the case of bilinear systems of difference equations.

The corresponding close-to-symmetric system (a recently introduced terminology, see, e.g, [58]) to equation (8) is

$$
z_{n+1}=\frac{\alpha w_{n}+\beta}{\gamma w_{n}+\delta}, \quad w_{n+1}=\frac{a z_{n}+b}{c z_{n}+d}, \quad n \in \mathbb{N}_{0},
$$

where $\alpha, \beta, \gamma, \delta, a, b, c, d, z_{0}, w_{0} \in \mathbb{R}($ or $\in \mathbb{C})$.

From (54) we easily obtain

$$
\begin{array}{r}
z_{n+1}=\frac{(a \alpha+\beta c) z_{n-1}+\alpha b+\beta d}{(a \gamma+c \delta) z_{n-1}+b \gamma+d \delta} \\
w_{n+1}=\frac{(a \alpha+b \gamma) w_{n-1}+a \beta+b \delta}{(\alpha c+\gamma d) w_{n-1}+\beta c+d \delta}
\end{array}
$$

for $n \in \mathbb{N}$.

Hence, the sequences

$$
z_{n}^{(j)}:=z_{2 n+j}, \quad n \in \mathbb{N}_{0}, j=0,1,
$$

are two solutions to the equation

$$
\widetilde{z}_{n+1}=\frac{\widetilde{\alpha} \widetilde{z}_{n}+\widetilde{\beta}}{\widetilde{\gamma} z_{n}+\widetilde{\delta}}, \quad n \in \mathbb{N}_{0}
$$

where

$$
\widetilde{\alpha}=a \alpha+\beta c, \quad \widetilde{\beta}=\alpha b+\beta d, \quad \widetilde{\gamma}=a \gamma+c \delta, \quad \widetilde{\delta}=b \gamma+d \delta,
$$

while the sequences

$$
w_{n}^{(j)}:=w_{2 n+j}, \quad n \in \mathbb{N}_{0}, j=0,1,
$$

are two solutions to the equation

$$
\widetilde{w}_{n+1}=\frac{\widehat{\alpha} \widetilde{w}_{n}+\widehat{\beta}}{\widehat{\gamma} \widetilde{w}_{n}+\widehat{\delta}}, \quad n \in \mathbb{N}_{0}
$$

where

$$
\widehat{\alpha}=a \alpha+b \gamma, \quad \widehat{\beta}=a \beta+b \delta, \quad \widehat{\gamma}=\alpha c+\gamma d, \quad \widehat{\delta}=\beta c+d \delta .
$$

For the case of bilinear equations (55) and (57), the corresponding equations in (26) are the same and are given by

$$
s_{n+1}-(a \alpha+b \gamma+c \beta+d \delta) s_{n}+(a d-b c)(\alpha \delta-\beta \gamma) s_{n-1}=0
$$

for $n \in \mathbb{N}_{0}$. 
It is not difficult to see that

$$
a \alpha+b \gamma+c \beta+d \delta=\widetilde{\alpha}+\widetilde{\delta}=\widehat{\alpha}+\widehat{\delta}
$$

and

$$
(a d-b c)(\alpha \delta-\beta \gamma)=\widetilde{\alpha} \widetilde{\delta}-\widetilde{\beta} \widetilde{\gamma}=\widehat{\alpha} \widehat{\delta}-\widehat{\beta} \widehat{\gamma} .
$$

From (60) and (61), we see that (59) can be written in the following two forms:

$$
s_{n+1}-(\widetilde{\alpha}+\widetilde{\delta}) s_{n}+(\widetilde{\alpha} \tilde{\delta}-\widetilde{\beta} \widetilde{\gamma}) s_{n-1}=0, \quad n \in \mathbb{N}_{0},
$$

and

$$
s_{n+1}-(\widehat{\alpha}+\widehat{\delta}) s_{n}+(\widehat{\alpha} \widehat{\delta}-\widehat{\beta} \widehat{\gamma}) s_{n-1}=0, \quad n \in \mathbb{N}_{0}
$$

which are the corresponding equations in (26) associated to equations (55) and (57), respectively.

By using Theorem 4, equations (62) and (63), as well as the following two equalities:

$$
z_{1}=\frac{\alpha w_{0}+\beta}{\gamma w_{0}+\delta} \quad \text { and } \quad w_{1}=\frac{a z_{0}+b}{c z_{0}+d}
$$

we obtain the following theorem.

Theorem 5 Consider system (54) with $(\alpha \delta-\beta \gamma)(a d-b c) \neq 0$. Assume that $\vec{v}=\left(v_{0}, v_{1}\right) \in \mathbb{R}^{2}$ $\left(\right.$ or $\left.\in \mathbb{C}^{2}\right)$ satisfies the condition

$$
(\beta \gamma-\alpha \delta)(a d-b c) v_{0}^{2}+(a \alpha+b \gamma+c \beta+d \delta) v_{0} v_{1} \neq v_{1}^{2} .
$$

Let $\left(s_{n}\right)_{n \in \mathbb{N}_{0}}$ be the solution to equation (59) such that $s_{0}=v_{0}$ and $s_{1}=v_{1}$. Then every welldefined solution $\left(z_{n}, w_{n}\right)_{n \in \mathbb{N}_{0}}$ to system (54) has the following representation:

$$
\begin{aligned}
z_{2 n}= & \frac{(\widetilde{\beta} \widetilde{\gamma}-\widetilde{\alpha} \widetilde{\delta})\left(\widetilde{\beta} v_{0}+\left(\widetilde{\alpha} v_{0}-v_{1}\right) z_{0}\right) s_{n-1}+\left(\widetilde{\beta}\left((\widetilde{\alpha}+\widetilde{\delta}) v_{0}-v_{1}\right)+z_{0}\left(v_{0}\left(\widetilde{\beta} \widetilde{\gamma}+\widetilde{\alpha}^{2}\right)-\widetilde{\alpha} v_{1}\right)\right) s_{n}}{\left((\widetilde{\beta} \widetilde{\gamma}-\widetilde{\alpha} \widetilde{\delta}) v_{0}+\widetilde{\alpha} v_{1}-\widetilde{\gamma} v_{1} z_{0}\right) s_{n}+\left(\widetilde{\delta} v_{0}-v_{1}+\widetilde{\gamma} v_{0} z_{0}\right) s_{n+1}}, \\
z_{2 n+1}= & \frac{(\widetilde{\beta} \widetilde{\gamma}-\widetilde{\alpha} \widetilde{\delta})\left(\widetilde{\beta} v_{0}\left(\gamma w_{0}+\delta\right)+\left(\widetilde{\alpha} v_{0}-v_{1}\right)\left(\alpha w_{0}+\beta\right)\right) s_{n-1}}{\left(\left((\widetilde{\beta} \widetilde{\gamma}-\widetilde{\alpha} \widetilde{\delta}) v_{0}+\widetilde{\alpha} v_{1}\right)\left(\gamma w_{0}+\delta\right)-\widetilde{\gamma} v_{1}\left(\alpha w_{0}+\beta\right)\right) s_{n}+\left(\left(\widetilde{\delta} v_{0}-v_{1}\right)\left(\gamma w_{0}+\delta\right)+\widetilde{\gamma} v_{0}\left(\alpha w_{0}+\beta\right)\right) s_{n+1}} \\
& +\frac{\left(\widetilde{\beta}\left((\widetilde{\alpha}+\widetilde{\delta}) v_{0}-v_{1}\right)\left(\gamma w_{0}+\delta\right)+\left(v_{0}\left(\widetilde{\beta} \widetilde{\gamma}+\widetilde{\alpha}^{2}\right)-\widetilde{\alpha} v_{1}\right)\left(\alpha w_{0}+\beta\right)\right) s_{n}}{\left(\left((\widetilde{\beta} \widetilde{\gamma}-\widetilde{\alpha} \widetilde{\delta}) v_{0}+\widetilde{\alpha} v_{1}\right)\left(\gamma w_{0}+\delta\right)-\widetilde{\gamma} v_{1}\left(\alpha w_{0}+\beta\right)\right) s_{n}+\left(\left(\widetilde{\delta} v_{0}-v_{1}\right)\left(\gamma w_{0}+\delta\right)+\widetilde{\gamma} v_{0}\left(\alpha w_{0}+\beta\right)\right) s_{n+1}}, \\
w_{2 n}= & \frac{(\widehat{\beta} \widehat{\gamma}-\widehat{\alpha} \widehat{\delta})\left(\widehat{\beta} v_{0}+\left(\widehat{\alpha} v_{0}-v_{1}\right) w_{0}\right) s_{n-1}+\left(\widehat{\beta}\left((\widehat{\alpha}+\widehat{\delta}) v_{0}-v_{1}\right)+w_{0}\left(v_{0}\left(\widehat{\beta} \widehat{\gamma}+\widehat{\alpha}^{2}\right)-\widehat{\alpha} v_{1}\right)\right) s_{n}}{\left((\widehat{\beta} \widehat{\gamma}-\widehat{\alpha} \widehat{\delta}) v_{0}+\widehat{\alpha} v_{1}-\widehat{\gamma} v_{1} w_{0}\right) s_{n}+\left(\widehat{\delta} v_{0}-v_{1}+\widehat{\gamma} v_{0} w_{0}\right) s_{n+1}}, \\
w_{2 n+1}= & \frac{(\widehat{\beta} \widehat{\gamma}-\widehat{\alpha} \widehat{\delta})\left(\widehat{\beta} v_{0}\left(c z_{0}+d\right)+\left(\widehat{\alpha} v_{0}-v_{1}\right)\left(a z_{0}+b\right)\right) s_{n-1}}{\left(\left((\widehat{\beta} \widehat{\gamma}-\widehat{\alpha} \widehat{\delta}) v_{0}+\widehat{\alpha} v_{1}\right)\left(c z_{0}+d\right)-\widehat{\gamma} v_{1}\left(a z_{0}+b\right)\right) s_{n}+\left(\left(\widehat{\delta} v_{0}-v_{1}\right)\left(c z_{0}+d\right)+\widehat{\gamma} v_{0}\left(a z_{0}+b\right)\right) s_{n+1}} \\
& +\frac{\left(\widehat{\beta}\left((\widehat{\alpha}+\widehat{\delta}) v_{0}-v_{1}\right)\left(c z_{0}+d\right)+\left(v_{0}\left(\widehat{\beta} \widehat{\gamma}+\widehat{\alpha}^{2}\right)-\widehat{\alpha} v_{1}\right)\left(a z_{0}+b\right)\right) s_{n}}{\left(\left((\widehat{\beta} \widehat{\gamma}-\widehat{\alpha} \widehat{\delta}) v_{0}+\widehat{\alpha} v_{1}\right)\left(c z_{0}+d\right)-\widehat{\gamma} v_{1}\left(a z_{0}+b\right)\right) s_{n}+\left(\left(\widehat{\delta} v_{0}-v_{1}\right)\left(c z_{0}+d\right)+\widehat{\gamma} v_{0}\left(a z_{0}+b\right)\right) s_{n+1}}
\end{aligned}
$$

$n \in \mathbb{N}_{0}$, where $\widetilde{\alpha}, \widetilde{\beta}, \widetilde{\gamma}, \widetilde{\delta}, \widehat{\alpha}, \widehat{\beta}, \widehat{\gamma}, \widehat{\delta}$ are defined in (56) and (58). 


\subsection{On a three-dimensional generalization of equation (8)}

Here we show that it is also possible to generalize Theorem 4 for the case of a threedimensional close-to-cyclic system of bilinear difference equations which extends difference equation (8).

The following three-dimensional close-to-cyclic system of bilinear difference equations is a natural generalization of difference equation (8), as well as of system of equations (54)

$$
z_{n+1}=\frac{a w_{n}+b}{c w_{n}+d}, \quad w_{n+1}=\frac{e u_{n}+f}{g u_{n}+h}, \quad u_{n+1}=\frac{p z_{n}+q}{r z_{n}+s}, \quad n \in \mathbb{N}_{0} \text {, }
$$

where $a, b, c, d, e, f, g, h, p, q, r, s, z_{0}, w_{0}, u_{0} \in \mathbb{R}($ or $\in \mathbb{C})$.

By using the method applied to system (54), it is easy to see that from the equations in (65) it follows that

$$
\begin{aligned}
& z_{n+1}=\frac{(a e p+b g p+a f r+b h r) z_{n-2}+a e q+b g q+a f s+b h s}{(c e p+d g p+c f r+d h r) z_{n-2}+c e q+d g q+c f s+d h s}, \\
& w_{n+1}=\frac{(a e p+a f r+c e q+c f s) w_{n-2}+b e p+b f r+d e q+d f s}{(a g p+a h r+c g q+c h s) w_{n-2}+b g p+b h r+d g q+d h s}, \\
& u_{n+1}=\frac{(p a e+q c e+p b g+q d g) w_{n-2}+p a f+q c f+p b h+q d h}{(r a e+s c e+r b g+s d g) w_{n-2}+r a f+s c f+r b h+s d h}
\end{aligned}
$$

for $n \geq 2$.

Hence, the sequences

$$
z_{n}^{(j)}:=z_{3 n+j}, \quad n \in \mathbb{N}_{0}, j=0,1,2,
$$

are three solutions to the equation

$$
\widetilde{z}_{n+1}=\frac{\widetilde{a} \widetilde{z}_{n}+\widetilde{b}}{\widetilde{c} \widetilde{z}_{n}+\widetilde{d}}, \quad n \in \mathbb{N}_{0}
$$

where

$$
\begin{aligned}
& \widetilde{a}=a e p+b g p+a f r+b h r \\
& \widetilde{b}=a e q+b g q+a f s+b h s, \\
& \widetilde{c}=c e p+d g p+c f r+d h r \\
& \widetilde{d}=c e q+d g q+c f s+d h s
\end{aligned}
$$

the sequences

$$
w_{n}^{(j)}:=w_{3 n+j}, \quad n \in \mathbb{N}_{0}, j=0,1,2,
$$

are three solutions to the equation

$$
\widetilde{w}_{n+1}=\frac{\widetilde{e} \widetilde{w}_{n}+\widetilde{f}}{\widetilde{g} \widetilde{w}_{n}+\widetilde{h}}, \quad n \in \mathbb{N}_{0}
$$


where

$$
\begin{aligned}
& \widetilde{e}=a e p+a f r+c e q+c f s, \\
& \widetilde{f}=b e p+b f r+d e q+d f s, \\
& \widetilde{g}=a g p+a h r+c g q+c h s, \\
& \widetilde{h}=b g p+b h r+d g q+d h s,
\end{aligned}
$$

while the sequences

$$
u_{n}^{(j)}:=u_{3 n+j}, \quad n \in \mathbb{N}_{0}, j=0,1,2,
$$

are three solutions to the equation

$$
\widetilde{u}_{n+1}=\frac{\widetilde{p} \widetilde{u}_{n}+\widetilde{q}}{\widetilde{r} \tilde{u}_{n}+\widetilde{s}}, \quad n \in \mathbb{N}_{0}
$$

where

$$
\begin{aligned}
& \widetilde{p}=p a e+q c e+p b g+q d g, \\
& \widetilde{q}=p a f+q c f+p b h+q d h, \\
& \widetilde{r}=r a e+s c e+r b g+s d g, \\
& \widetilde{s}=r a f+s c f+r b h+s d h .
\end{aligned}
$$

For the case of bilinear equations (66), (71), and (76), the corresponding equations in (26) are the same and are given by

$$
\begin{aligned}
& s_{n+1}-(a e p+b g p+a f r+b h r+c e q+d g q+c f s+d h s) s_{n} \\
& +(a d-b c)(e h-f g)(p s-q r) s_{n-1}=0
\end{aligned}
$$

for $n \in \mathbb{N}_{0}$.

It is not difficult to see that

$$
a e p+b g p+a f r+b h r+c e q+d g q+c f s+d h s=\widetilde{a}+\widetilde{d}=\widetilde{e}+\widetilde{h}=\widetilde{p}+\widetilde{s}
$$

and

$$
(a d-b c)(e h-f g)(p s-q r)=\widetilde{a} \widetilde{d}-\widetilde{b} \widetilde{c}=\widetilde{e} \widetilde{h}-\widetilde{f} \widetilde{g}=\widetilde{p s}-\widetilde{q} \widetilde{r} .
$$

From (82) and (83), we see that (81) can be written in the following three forms:

$$
\begin{aligned}
& s_{n+1}-(\widetilde{a}+\widetilde{d}) s_{n}+(\widetilde{a} \tilde{d}-\widetilde{b} \widetilde{c}) s_{n-1}=0, \\
& s_{n+1}-(\widetilde{e}+\widetilde{h}) s_{n}+(\widetilde{e} \widetilde{h}-\widetilde{f} \widetilde{g}) s_{n-1}=0,
\end{aligned}
$$


and

$$
s_{n+1}-(\widetilde{p}+\widetilde{s}) s_{n}+(\widetilde{p} \widetilde{s}-\widetilde{q} \widetilde{r}) s_{n-1}=0
$$

for $n \in \mathbb{N}_{0}$, which are the corresponding equations in (26) associated to equations (66), (71), and (76), respectively.

Hence, by using Theorem 4, (84)-(86), and the following equalities:

$$
z_{1}=\frac{a w_{0}+b}{c w_{0}+d}, \quad w_{1}=\frac{e u_{0}+f}{g u_{0}+h}, \quad u_{1}=\frac{p z_{0}+q}{r z_{0}+s},
$$

we get the following result.

\section{Theorem 6 Consider system (65). Assume that}

$$
(a d-b c)(e h-f g)(p s-q r) \neq 0,
$$

$\vec{v}=\left(v_{0}, v_{1}\right) \in \mathbb{R}^{2}$ (or $\left.\in \mathbb{C}^{2}\right)$ satisfies the condition

$$
(b c-a d)(e h-f g)(p s-q r) v_{0}^{2}+(a e p+b g p+a f r+b h r+c e q+d g q+c f s+d h s) v_{0} v_{1} \neq v_{1}^{2} \text {. }
$$

Let $\left(s_{n}\right)_{n \in \mathbb{N}_{0}}$ be the solution to equation (81) such that $s_{0}=v_{0}$ and $s_{1}=v_{1}$. Then every welldefined solution $\left(z_{n}, w_{n}, u_{n}\right)_{n \in \mathbb{N}_{0}}$ to system (65) has the following representation:

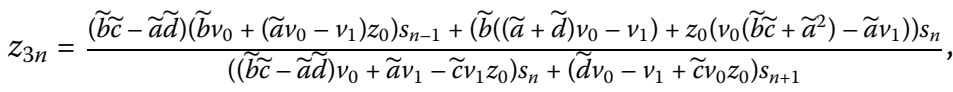

$$
\begin{aligned}
& w_{3 n}=\frac{\left.(\widetilde{f} \tilde{g}-\tilde{e} \widetilde{h})\left(\widetilde{f} v_{0}+\left(\widetilde{e} v_{0}-v_{1}\right) w_{0}\right) s_{n-1}+\left(\widetilde{f}(\widetilde{e}+\widetilde{h}) v_{0}-v_{1}\right)+w_{0}\left(v_{0}\left(\widetilde{f} \widetilde{g}+\widetilde{e}^{2}\right)-\widetilde{e} v_{1}\right)\right) s_{n}}{\left((\widetilde{f} \widetilde{g}-\widetilde{e} \tilde{h}) v_{0}+\widetilde{e} v_{1}-\widetilde{g} v_{1} w_{0}\right) s_{n}+\left(\widetilde{h} v_{0}-v_{1}+\widetilde{g} v_{0} w_{0}\right) s_{n+1}}, \\
& u_{3 n}=\frac{(\widetilde{q} \tilde{r}-\widetilde{p} s)\left(\widetilde{q} v_{0}+\left(\widetilde{p} v_{0}-v_{1}\right) u_{0}\right) s_{n-1}+\left(\widetilde{q}\left((\widetilde{p}+\widetilde{s}) v_{0}-v_{1}\right)+u_{0}\left(v_{0}\left(\widetilde{q} \widetilde{r}+\widetilde{p}^{2}\right)-\widetilde{p} v_{1}\right)\right) s_{n}}{\left((\widetilde{q} r-\widetilde{p}) v_{0}+\widetilde{p} v_{1}-\widetilde{r} v_{1} u_{0}\right) s_{n}+\left(\widetilde{s} v_{0}-v_{1}+\widetilde{r} v_{0} u_{0}\right) s_{n+1}}, \\
& z_{3 n+1}=\frac{(\tilde{b} \tilde{c}-\tilde{a} \tilde{d})\left(\tilde{b} v_{0}\left(c w_{0}+d\right)+\left(\widetilde{a} v_{0}-v_{1}\right)\left(a w_{0}+b\right)\right) s_{n-1}}{\left.\left((\tilde{b} \tilde{c}-\tilde{a} \tilde{d}) v_{0}+\tilde{a} v_{1}\right)\left(c w_{0}+d\right)-\tilde{c} v_{1}\left(a w_{0}+b\right)\right) s_{n}+\left(\left(\tilde{d} v_{0}-v_{1}\right)\left(c w_{0}+d\right)+\widetilde{c} v_{0}\left(a w_{0}+b\right)\right) s_{n+1}} \\
& +\frac{\left(\widetilde{b}\left((\widetilde{a}+\widetilde{d}) v_{0}-v_{1}\right)\left(c w_{0}+d\right)+\left(v_{0}\left(\widetilde{b} \widetilde{c}+\widetilde{a}^{2}\right)-\widetilde{a} v_{1}\right)\left(a w_{0}+b\right)\right) s_{n}}{\left(\left((\tilde{b} \widetilde{c}-\tilde{a} \tilde{d}) v_{0}+\widetilde{a} v_{1}\right)\left(c w_{0}+d\right)-\widetilde{c} v_{1}\left(a w_{0}+b\right)\right) s_{n}+\left(\left(\widetilde{d} v_{0}-v_{1}\right)\left(c w_{0}+d\right)+\widetilde{c} v_{0}\left(a w_{0}+b\right)\right) s_{n+1}}, \\
& w_{3 n+1}=\frac{\tilde{f} \widetilde{g}-\widetilde{e} \tilde{h})\left(\tilde{f} v_{0}\left(g u_{0}+h\right)+\left(\tilde{e} v_{0}-v_{1}\right)\left(e u_{0}+f\right)\right) s_{n-1}}{\left(\left((\widetilde{f} \tilde{g}-\widetilde{e} \tilde{h}) v_{0}+\widetilde{e} v_{1}\right)\left(g u_{0}+h\right)-\widetilde{g} v_{1}\left(e u_{0}+f\right)\right) s_{n}+\left(\left(\widetilde{h} v_{0}-v_{1}\right)\left(g u_{0}+h\right)+\widetilde{g} v_{0}\left(e u_{0}+f\right)\right) s_{n+1}} \\
& \left.\widetilde{f}\left((\widetilde{e}+\widetilde{h}) v_{0}-v_{1}\right)\left(g u_{0}+h\right)+\left(v_{0}\left(\widetilde{f} \widetilde{g}+\widetilde{e}^{2}\right)-\widetilde{e} v_{1}\right)\left(e u_{0}+f\right)\right) s_{n} \\
& +\frac{\left.\left.(\widetilde{f} \widetilde{g}-\widetilde{e} \tilde{h}) v_{0}+\widetilde{e} v_{1}\right)\left(g u_{0}+h\right)-\widetilde{g} v_{1}\left(e u_{0}+f\right)\right) s_{n}+\left(\left(\widetilde{h} v_{0}-v_{1}\right)\left(g u_{0}+h\right)+\widetilde{g} v_{0}\left(e u_{0}+f\right)\right) s_{n+1}}{,}, \\
& (\widetilde{q} \widetilde{r}-\widetilde{p} \widetilde{s})\left(\widetilde{q} v_{0}\left(r z_{0}+s\right)+\left(\widetilde{p} v_{0}-v_{1}\right)\left(p z_{0}+q\right)\right) s_{n-1} \\
& u_{3 n+1}=\frac{\left.\left.(\widetilde{q} \tilde{r}-\widetilde{p}) v_{0}+\widetilde{p} v_{1}\right)\left(r z_{0}+s\right)-\widetilde{r} v_{1}\left(p z_{0}+q\right)\right) s_{n}+\left(\left(\widetilde{s} v_{0}-v_{1}\right)\left(r z_{0}+s\right)+\widetilde{r} v_{0}\left(p z_{0}+q\right)\right) s_{n+1}}{((\widetilde{q})} \\
& \left(\widetilde{q}\left((\widetilde{p}+\widetilde{s}) v_{0}-v_{1}\right)\left(r z_{0}+s\right)+\left(v_{0}\left(\widetilde{q} \widetilde{r}+\widetilde{p}^{2}\right)-\widetilde{p} v_{1}\right)\left(p z_{0}+q\right)\right) s_{n} \\
& +\frac{\left.\widetilde{q}\left((\widetilde{q}-\widetilde{p}) v_{0}+\widetilde{p} v_{1}\right)\left(r z_{0}+s\right)-\widetilde{r} v_{1}\left(p z_{0}+q\right)\right) s_{n}+\left(\left(\widetilde{s} v_{0}-v_{1}\right)\left(r z_{0}+s\right)+\widetilde{r} v_{0}\left(p z_{0}+q\right)\right) s_{n+1}}{(\widetilde{h}} \\
& z_{3 n+2}=\frac{(\widetilde{b} \widetilde{c}-\tilde{a} \widetilde{d})\left(\widetilde{b} v_{0}+\left(\widetilde{a} v_{0}-v_{1}\right) z_{2}\right) s_{n-1}+\left(\widetilde{b}\left((\widetilde{a}+\widetilde{d}) v_{0}-v_{1}\right)+z_{2}\left(v_{0}\left(\widetilde{b} \widetilde{c}+\widetilde{a}^{2}\right)-\widetilde{a} v_{1}\right)\right) s_{n}}{\left((\widetilde{b} \widetilde{c}-\tilde{a} \tilde{d}) v_{0}+\widetilde{a} v_{1}-\widetilde{c} v_{1} z_{2}\right) s_{n}+\left(\widetilde{d} v_{0}-v_{1}+\widetilde{c} v_{0} z_{2}\right) s_{n+1}},
\end{aligned}
$$

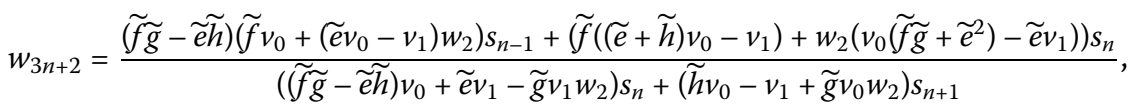

$$
\begin{aligned}
& u_{3 n+2}=\frac{(\widetilde{q} \widetilde{r}-\widetilde{p} \widetilde{s})\left(\widetilde{q} v_{0}+\left(\widetilde{p} v_{0}-v_{1}\right) u_{2}\right) s_{n-1}+\left(\widetilde{q}\left((\widetilde{p}+\widetilde{s}) v_{0}-v_{1}\right)+u_{2}\left(v_{0}\left(\widetilde{q} \tilde{r}+\widetilde{p}^{2}\right)-\widetilde{p} v_{1}\right)\right) s_{n}}{\left((\widetilde{q} r-\widetilde{p} s) v_{0}+\widetilde{p} v_{1}-\widetilde{r} v_{1} u_{2}\right) s_{n}+\left(\widetilde{s} v_{0}-v_{1}+\widetilde{r} v_{0} u_{2}\right) s_{n+1}}
\end{aligned}
$$


for every $n \in \mathbb{N}_{0}$ and each $j=0,1,2$, where $\tilde{a}, \widetilde{b}, \widetilde{c}, \tilde{d}, \widetilde{e}, \widetilde{f}, \widetilde{g}, \widetilde{h}, \widetilde{p}, \widetilde{q}, \widetilde{r}, \widetilde{s}$ are defined by (67)-(70), (72)-(75), (77)-(80), respectively.

Remark 2 In order to get all the formulas in Theorem 6 in terms of initial values $z_{0}, w_{0}$, and $u_{0}$, in the formulas for $z_{3 n+2}, w_{3 n+2}$, and $u_{3 n+2}$, the following equalities should be used:

$$
\begin{aligned}
& z_{2}=\frac{(a e+b g) u_{0}+a f+b h}{(c e+d g) u_{0}+c f+d h}, \\
& w_{2}=\frac{(e p+f r) z_{0}+e q+f s}{(g p+h r) z_{0}+g q+h s}, \\
& u_{2}=\frac{(p a+q c) w_{0}+p b+q d}{(r a+s c) w_{0}+r b+s d},
\end{aligned}
$$

which follows from the equations in (65). We have not done this since such obtained formulas are so long that cannot be written in a line without a drastic reduction of letters. Hence, we leave the simple task to the interested reader.

Remark 3 It is time consuming but not difficult to check that the above procedure employed to the two-dimensional close-to-symmetric and three-dimensional close-to-cyclic bilinear systems of difference equations can be also applied to close-to-cyclic bilinear systems of difference equations of fourth order. However, since the procedure leads to some long calculations and quite long formulas, we also leave getting the corresponding formulas for solutions to the system to the interested reader.

\section{On the name of equation (8)}

As we have already mentioned, difference equations appeared in the form of recurrent relations/series in 1718 at the latest (before that time they usually had appeared, in a way, indirectly or descriptively). The methods by de Moivre were systematized and studied further by Euler and can be found in [7]. Later in 1759 Lagrange in [34] studied the "integrability" (i.e., solvability) of linear difference equations by modifying the methods which had been used in studying differential equations and essentially laid a cornerstone for further investigations.

There has been a recent custom that the bilinear difference equation is called Riccati difference equation. During the last two decades several colleagues, some of them who used the terminology, asked me if the attribution is correct, bearing in mind that it is known that there were not so many investigations on difference equations during the life of Riccati and that nobody has seen a paper written by him on the topic. The frequent question and recent studies of solvability of difference equations motivated me to conduct a considerable, but, of course, not thorough, literature investigation, to try to give a possible answer for the "open problem" in history of difference equations.

As far as we could see, the bilinear difference equation had not been investigated by Riccati at all, which is not so strange bearing in mind that he lived until 1754 and that a serious investigation of the solvability of difference equations practically started in Lagrange's 1759 paper [34].

Reputable sources from the end of eighteenth century, which are almost of an encyclopedic character, such us Cousin's book [63] (1796) and famous Lacroix book [64] (1800) did not mention Riccati in chapters devoted to difference equations at all. Moreover, Lacroix, 
besides already mentioned mathematicians and other French ones, mentioned in [64] several Italians, such as Brunacci, Paoli, and Malfatti, who had worked on difference equations. If we follow further the nineteenth century literature on difference equations all over Europe (see, for example, Lacroix [65] (1816), Schlömilch [66] (1848), Boole [20] (1880, 1st ed. 1860), Markoff [67, 68] (1896, 2nd ed. 1910)), we see that nobody mention Riccati at all. Although, for example, Boole devoted considerable space in his book to studying bilinear difference equations. It should be also mentioned that the elementary course on differential and integral calculus [65] by Lacroix (1816) considers the bilinear difference equation and solves it by using the change of variables (14), which means that already at the time the solvability of the equation was regarded as a matter of general mathematical culture, as something which is easily understandable. Nörlund in his known book [69] did not consider equation (8), but the book contains a huge number of references not mentioning Riccati at all.

The first encounter of any connection to the bilinear difference equation with Riccati's name, that we managed to find so far, is in book [24] by Milne-Thomson (1933). Namely, he called the following difference equation

$$
u(x) u(x+1)+p(x) u(x+1)+q(x) u(x)+r(x)=0
$$

with variable coefficients as "of Riccati's form" and only said that "it is a difference equation corresponding to Riccati's differential one". This means that he did not attribute the equation to Riccati but simply made an analogy, which is nowadays a well-known fact to any expert on difference equations. It should be also noticed that this part of his book seems to be essentially taken from Boole's book [20], which, as we have already mentioned, does not mention Riccati, at all. Whether or not Milne-Thomson is the first who used the terminology is not known to us, but bearing in mind that his book was one of a few books on difference equations at that time, it certainly had some influence in spreading it. It should be also noticed that other books on difference equations up to the 70s did not mention Riccati either (see, e.g., Fort [23] (1948), Richardson [27] (1954), Levy and Lessman [28] (1961)).

The cherry on the cake could be the classroom note [21] by Brand (1955) published in a widely read popular journal, which solves the bilinear difference equation in the way as it was suggested by old French masters $[6,65]$ and describes the long-term behavior of its solutions. The note starts with: "The sequences defined by a difference equation of Riccati type", but the note is devoted only to the bilinear difference equation, that is, to equation (8) (with constant coefficients). Interestingly [21] did not cite any paper except [16] because of the Kronecker lemma. Bearing in mind that the terminology is similar to the one in [24], we can suppose that Brand borrowed it from there. As we can see, Brand also did not attribute the bilinear difference equation to Riccati, but it seems this, combined with book [24], caused the chain reaction among some experts who started calling equation (8) by a wrong name, that is, a Riccati equation.

Remark 4 Recall that there has been a related problem with solution (2) to equation (1), which has been frequently called Binet's solution (we personally met the attribution for the first time in a Russian issue of book [12], which could have been simply another case of a wrong chain reaction). Nowadays it is well known that, in fact, formula (2) belongs to 
de Moivre. The formula and the method that leads to getting it can be also regarded as a cornerstone in the study of solvability of difference equations (one of several cornerstones by de Moivre, besides the formula $(\cos \varphi+i \sin \varphi)^{n}=\cos n \varphi+i \sin n \varphi$, and de MoivreLaplace theorem).

\section{Acknowledgements}

The study in the paper is a part of the investigation under the projects III 41025 and III 44006 by the Serbian Ministry of Education and Science.

\section{Funding}

Not applicable.

Availability of data and materials

Not applicable.

\section{Competing interests}

The author declares that he has no competing interests.

\section{Authors' contributions}

The author has contributed solely to the writing of this paper. He read and approved the manuscript.

\section{Author details}

${ }^{1}$ Mathematical Institute of the Serbian Academy of Sciences, Beograd, Serbia. ${ }^{2}$ Department of Medical Research, China Medical University Hospital, China Medical University, Taichung, Taiwan, Republic of China. ${ }^{3}$ Department of Healthcare Administration, Asia University, Taichung, Taiwan, Republic of China.

\section{Publisher's Note}

Springer Nature remains neutral with regard to jurisdictional claims in published maps and institutional affiliations.

Received: 10 October 2018 Accepted: 11 December 2018 Published online: 29 December 2018

\section{References}

1. de Moivre, A.: De Fractionibus algebraicis radicalitate immunibus ad fractiones simpliciores reducendis, deque summandis terminis quarumdam serierum aequali intervallo a se distantibus. Philos. Trans. 32, 162-178 (1722) (in Latin)

2. de Moivre, A.: The Doctrine of Chances. London (1718)

3. de Moivre, A.: Miscellanea analytica de seriebus et quadraturis. Londini (1730) (in Latin)

4. de Moivre, A.: The Doctrine of Chances, 3rd edn. Strand, London (1756)

5. Fibonacci, L.: Liber Abbaci (1202) (in Latin)

6. Laplace, P.S.: Recherches sur l'intégration des équations différentielles aux différences finies et sur leur usage dans la théorie des hasards. Mémoires de I' Académie Royale des Sciences de Paris 1773, t. VII (1776) (Laplace OEuvres, VIII, 69-197, 1891) (in French)

7. Eulero, L.: Introductio in Analysin Infinitorum. Tomus Primus, Lausannae (1748) (in Latin)

8. Stević, S.: Representation of solutions of bilinear difference equations in terms of generalized Fibonacci sequences. Electron. J. Qual. Theory Differ. Equ. 2014, Article ID 67 (2014)

9. Stević, S.: First-order product-type systems of difference equations solvable in closed form. Electron. J. Differ. Equ. 2015, Article ID 308 (2015)

10. Stević, S., Iričanin, B., Šmarda, Z:: Two-dimensional product-type system of difference equations solvable in closed form. Adv. Differ. Equ. 2016, Article ID 253 (2016)

11. Alfred, B.U.: An Introduction to Fibonacci Discovery. The Fibonacci Association (1965)

12. Vorobiev, N.N.: Fibonacci Numbers. Birkhäuser, Basel (2002) (Russian first edition 1950)

13. Lagrange, J.-L.: OEuvres, t. II. Gauthier-Villars, Paris (1868) (in French)

14. Möbius, A.F.: Veber involutionen höherer Ordnung. Leipz. Sitzungsber. Math.-Phys. 7, 123-140 (1855) (in German)

15. Krechmar, V.A.: A Problem Book in Algebra. Mir, Moscow (1974) (Russian first edition 1937)

16. Hardy, G.H., Wright, E.M.: An Introduction to the Theory of Numbers. Clarendon Press, Oxford (1938)

17. Adamović, D.: Problem 194. Mat. Vesn. 22(2), 270 (1970)

18. Adamović, D.: Solution to problem 194. Mat. Vesn. 23, 236-242 (1971)

19. Agarwal, R.P.: Difference Equations and Inequalities: Theory, Methods, and Applications, 2nd edn. Dekker, New York (2000)

20. Boole, G.: A Treatise on the Calculus of Finite Differences, 3rd edn. Macmillan \& Co., London (1880)

21. Brand, L: A sequence defined by a difference equation. Am. Math. Mon. 62(7), 489-492 (1955)

22. Brand, L.: Differential and Difference Equations. Wiley, New York (1966)

23. Fort, T.: Finite Differences and Difference Equations in the Real Domain. Clarendion Press, Oxford (1948)

24. Milne-Thomson, L.M.: The Calculus of Finite Differences. MacMillan \& Co., London (1933)

25. Mitrinović, D.S., Adamović, D.D.: Sequences and Series. Naučna Knjiga, Beograd (1980) (In Serbian)

26. Mitrinović, D.S., Kečkić, J.D.: Methods for Calculating Finite Sums. Naučna Knjiga, Beograd (1984) (in Serbian)

27. Richardson, C.H.: An Introduction to the Calculus of Finite Differences. Van Nostrand, New York (1954)

28. Levy, H., Lessman, F.: Finite Difference Equations. Dover, New York (1992) (Original version 1961) 
29. Stević, S.: Solutions of a max-type system of difference equations. Appl. Math. Comput. 218, 9825-9830 (2012)

30. Stević, S.: A four-dimensional solvable system of difference equations in the complex domain. Rev. R. Acad. Cienc. Exactas Fís. Nat., Ser. A Mat. 112(4), 1265-1280 (2018)

31. Berezansky, L., Braverman, E.: On impulsive Beverton-Holt difference equations and their applications. J. Differ. Equ. Appl. 10(9), 851-868 (2004)

32. Berg, L., Stević, S.: On some systems of difference equations. Appl. Math. Comput. 218, 1713-1718 (2011)

33. Stević, S.: On some solvable systems of difference equations. Appl. Math. Comput. 218, 5010-5018 (2012)

34. Lagrange, J.-L.: Sur l'intégration d'une équation différentielle à différences finies, qui contient la théorie des suites récurrentes. Miscellanea Taurinensia, t. I, (1759), 33-42 (Lagrange OEuvres, I, 23-36, 1867) (in French)

35. Jordan, C.: Calculus of Finite Differences. Chelsea, New York (1956)

36. Papaschinopoulos, G., Stefanidou, G.: Asymptotic behavior of the solutions of a class of rational difference equations. Int. J. Difference Equ. 5(2), 233-249 (2010)

37. Stević, S.: On the difference equation $x_{n}=x_{n-2} /\left(b_{n}+c_{n} x_{n-1} x_{n-2}\right)$. Appl. Math. Comput. 218, 4507-4513 (2011)

38. Stević, S:: On the difference equation $x_{n}=x_{n-k} /\left(b+c x_{n-1} \cdots x_{n-k}\right)$. Appl. Math. Comput. 218, 6291-6296 (2012)

39. Stević, S.: Solvable subclasses of a class of nonlinear second-order difference equations. Adv. Nonlinear Anal. 5(2), 147-165 (2016)

40. Stević, S., Diblik, J., Iričanin, B., Šmarda, Z.: On some solvable difference equations and systems of difference equations. Abstr. Appl. Anal. 2012, Article ID 541761 (2012)

41. Stević, S., Diblik, J., Iričanin, B., Šmarda, Z:: On the difference equation $x_{n}=a_{n} x_{n-k} /\left(b_{n}+c_{n} x_{n-1} \cdots x_{n-k}\right)$. Abstr. Appl. Anal. 2012, Article ID 409237 (2012)

42. Stević, S., Diblik, J., Iričanin, B., Šmarda, Z.: On a solvable system of rational difference equations. J. Differ. Equ. Appl. 20(5-6), 811-825 (2014)

43. Andruch-Sobilo, A., Migda, M.: Further properties of the rational recursive sequence $x_{n+1}=a x_{n-1} /\left(b+c x_{n} x_{n-1}\right)$. Opusc. Math. 26(3), 387-394 (2006)

44. Andruch-Sobilo, A., Migda, M.: On the rational recursive sequence $x_{n+1}=a x_{n-1} /\left(b+c x_{n} x_{n-1}\right)$. Tatra Mt. Math. Publ. 43, 1-9 (2009)

45. Agarwal, R.P., Popenda, J.: Periodic solutions of first order linear difference equations. Math. Comput. Model. 22(1), $11-19(1995)$

46. Stević, S.: Bounded and periodic solutions to the linear first-order difference equation on the integer domain. Adv. Differ. Equ. 2017, Article ID 283 (2017)

47. Stević, S.: Bounded solutions to nonhomogeneous linear second-order difference equations. Symmetry 9, Article ID $227(2017)$

48. Iričanin, B., Stević, S.: Eventually constant solutions of a rational difference equation. Appl. Math. Comput. 215 854-856 (2009)

49. Mitrinović, D.S.: Mathematical Induction, Binomial Formula, Combinatorics. Gradjevinska Knjiga, Beograd (1980) (in Serbian)

50. Mitrinović, D.S.: Matrices and Determinants. Naučna Knjiga, Beograd (1989) (in Serbian)

51. Papaschinopoulos, G., Schinas, C.J.: Invariants for systems of two nonlinear difference equations. Differ. Equ. Dyn. Syst. 7, 181-196 (1999)

52. Papaschinopoulos, G., Schinas, C.J.: Invariants and oscillation for systems of two nonlinear difference equations. Nonlinear Anal., Theory Methods Appl. 46, 967-978 (2001)

53. Papaschinopoulos, G., Schinas, C.J., Stefanidou, G.: On a k-order system of Lyness-type difference equations. Adv. Differ. Equ. 2007, Article ID 31272 (2007)

54. Stević, S.: On a generalized max-type difference equation from automatic control theory. Nonlinear Anal. TMA 72 , 1841-1849 (2010)

55. Stević, S.: On the system $x_{n+1}=y_{n} x_{n-k} /\left(y_{n-k+1}\left(a_{n}+b_{n} y_{n} x_{n-k}\right)\right), y_{n+1}=x_{n} y_{n-k} /\left(x_{n-k+1}\left(c_{n}+d_{n} x_{n} y_{n-k}\right)\right)$. Appl. Math. Comput. 219, 4526-4534 (2013)

56. Stević, S.: Note on the binomial partial difference equation. Electron. J. Qual. Theory Differ. Equ. 2015, Article ID 96 (2015)

57. Stević, S.: Existence of a unique bounded solution to a linear second order difference equation and the linear first order difference equation. Adv. Differ. Equ. 2017, Article ID 169 (2017)

58. Stević, S., Iričanin, B., Šmarda, Z.: On a close to symmetric system of difference equations of second order. Adv. Differ. Equ. 2015, Article ID 264 (2015)

59. Tollu, D.T., Yazlik, Y., Taskara, N.: On the solutions of two special types of Riccati difference equation via Fibonacci numbers. Adv. Differ. Equ. 2013, Article ID 174 (2013)

60. (Group of authors) Collection of Solved Problems from Competitions of Young Serbian Mathematicians in 1983. Serbian Mathematical Society (1983) (in Serbian)

61. Ašić, M., et al.: Federal and Republic Secondary School Competitions in Mathematics. Serbian Mathematical Society, Belgrade (1984) (in Serbian)

62. Stević, S., Iričanin, B., Kosmala, W., Šmarda, Z.: Note on the bilinear difference equation with a delay. Math. Methods Appl. Sci. 41, 9349-9360 (2018)

63. Cousin, J.A.J.: Traité de Calcul Différentiel et de Calcul Intégral. Régent et Bernard, Paris (1796) (in French)

64. Lacroix, S.F.: Traité des Differénces et des Séries. J.B.M. Duprat, Paris (1800) (in French)

65. Lacroix, S.F.: An Elementary Treatise on the Differential and Integral Calculus. W.P. Grant, Cambridge (1816)

66. Schlömilch, O.: Theorie der Differenzen und Summen. Druck und Verlag H. W. Schmidt, Halle (1848) (in German)

67. Markoff, A.A.: Differenzenrechnung. Teubner, Leipzig (1896) (in German)

68. Markov, A.A.: Ischislenie Konechnykh Raznostey, 2nd edn. Matezis, Odessa (1910) (in Russian)

69. Nörlund, N.E.: Vorlesungen Über Differenzenrechnung. Springer, Berlin (1924) (in German) 